\title{
论文
}

\section{聚磷酸酯阻燃剂复配蒙脱土及聚磷酸铵对环氧树 脂阻燃性能的影响}

\author{
杨吉, 张永航, 范娟娟, 闵样, 班大明“ \\ 贵州师范大学化学与材料科学学院, 贵阳 550025 \\ *通讯作者, E-mail: bdaming@gznu.edu.cn \\ 收稿日期: 2019-10-20; 接受日期: 2020-02-10; 网络版发表日期: 2020-03-25 \\ 国家自然科学基金(编号: 51763005)、贵州省科技厅(黔科合人才[2017]5726-31)资助项目
}

摘要本文以二氯化磷酸对甲基苯酯和 10-(2,5-二羟基苯基)-10-氢-9-氧杂-10-磷杂菲-10-氧化物(ODOPB)为原 料, 合成了一种新型聚磷酸酯阻燃剂聚磷酸-2-10-氢-9-氧杂-10-磷杂菲-10-氧化物基对苯二酚对甲苯酯(POTP), 并 采用傅里叶变换红外光谱(FTIR)和核磁共振( ${ }^{31} \mathrm{P}-\mathrm{NMR},{ }^{1} \mathrm{H}-\mathrm{NMR}$ 和 $\left.{ }^{13} \mathrm{C}-\mathrm{NMR}\right)$ 对其结构进行表征. 将POTP与蒙脱 土(MMT) 及聚磷酸铵 (APP) 组成复合阻燃剂对环氧树脂 (EP) 进行阻燃改性, 通过垂直燃烧(UL-94)、氧指数 (LOI)、热失重(TGA)、锥形量热(CONE) 和扫描电镜(SEM)等方法研究其对EP的热性能和阻燃性能的影响. 结果 表明, 当阻燃剂添加量为 7\% 时, EP复合材料UL-94测试等级可达V-0级; 当添加阻燃剂为 $9 \%$ 时, 其 LOI值可达到 $27.6 \%$, 最大热释放速率(Pk-HRR)下降了 $50.1 \%$, 热释放总量(THR)下降了 $27.4 \%$, 其残炭量高达 $29 \%$. CONE测试后 的残炭形貌研究显示阻燃 $\mathrm{EP}$ 在高温下形成较稳定的致密膨胀炭层, 能有效抑制烟毒性气体释放, 隔绝可燃气体与 空气的交换, 从而提高阻燃EP在高温下的热稳定性和阻燃性能.

关键词环氧树脂, 蒙脱土, 聚磷酸铵, 阻燃性能

\section{1 引言}

环氧树脂(EP)是一种重要的热固性树脂，具有优 良的物理、化学特性及良好的加工性能，被广泛应用 于电子电器绝缘、航空航天及半导体密封剂等诸多领 域 ${ }^{[1 ~ 3]}$. 由于EP极易燃烧, 且在燃烧过程释放大量的毒 气和烟雾, 所以在很大程度上限制了其应用, 因此对阻 燃EP的研究具有重要意义.

传统的卤素阻燃剂虽然具有较好的阻燃效果，但 是由于在其燃烧过程中会释放大量的有毒及腐蚀性气
体, 严重危害到人类的健康, 因此无卤阻燃剂的研究越 来越受到人们的重视 ${ }^{[4,5]}$. 有机磷系阻燃剂具有良好的 阻燃性能且较为绿色环保, 近年来, 通过加入含磷阻燃 剂如氧化膦、有机膦酸盐以及其它膦化衍生物等制备 阻燃环氧树脂的文献报道越来越多 ${ }^{[6-8]}$. 9,10-二氢-9氧杂-10-磷杂菲-10-氧化物(DOPO) 是一种新型磷系阻 燃剂中间体, 由于其具有良好热稳定性、耐氧化性及 阻燃性能、与环氧树脂较好的相容性等, 因此对 DOPO及其衍生物阻燃剂的研究备受人们关注 ${ }^{[9,10]}$. Perret等 ${ }^{[11]}$ 通过设计合成的星形脂肪族四羟乙基戊四

引用格式: Yang J, Zhang Y, Fan J, Min Y, Ban D. Effect of polyphosphate flame retardant compounded montmorillonite and ammonium polyphosphate on flame retardancy of epoxy resin. Sci Sin Chim, 2020, 50: 489-497, doi: 10.1360/SSC-2020-0010 
酯(DOPP)或杂环三羟乙基异氰尿酸酯(DOPI)对环氧 树脂进行阻燃改性, $5.9 \%$ 的DOPP和 $69.9 \%$ 的碳纤维复 配, 阻燃EP的UL-94测试达V-0级; DOPI添加量为 $23.1 \%$ 时，阻燃EP通过UL-94试验获得V-0评级. Zhang 等 ${ }^{[12]}$ 设计合成了一种低含磷量的DOPO衍生物 PBI 阻 燃剂改性环氧树脂，添加 $10.8 \%$ 的PBI通过UL-94试验 可以获得 $\mathrm{V}-0$ 评级. $\mathrm{Xu}$ 等 ${ }^{[13]}$ 合成了一种DOPO衍生物 CTP-DOPO用于改性环氧树脂，当CTP-DOPO的负载 量为 $10.6 \%$ 时，阻燃EP通过UL-94试验获得V-0评级. 但是这些工作存在阻燃剂结构复杂、制备工艺繁琐、 添加量较大而催化成炭能力较差的缺点.

因此，我们尝试合成一种结构简单、高含磷量的 新型磷酸酯类阻燃剂—聚磷酸-2-10-氢- 9 -氧杂- 10 -磷 杂菲-10-氧化物基对苯二酚对甲苯酯(POTP)，用来制 备综合性能优异、高阻燃效率的阻燃 $\mathrm{EP}$ 复合材料. 将 其与 $\mathrm{APP}$ (聚磷酸铵)复配加入到EP中，当阻燃剂加入 量为 $5 \%$ 时, 阻燃EP的UL-94测试即可达到 V-0级, 但 LOI值只有 $21.7 \%$; 阻燃剂加入量为 $10 \%$ 时，阻燃EP的 $\mathrm{LOI}$ 值也仅为 $22.9 \%$. 为了进一步提高其 $\mathrm{LOI}$ 值, 我们将 MMT(蒙脱土)引入到POTP/APP/EP复合材料中．通过 垂直燃烧、氧指数、热失重、雉形量热和扫描电镜等 手段分析研究表明，阻燃EP复合材料具有良好的阻燃 性能和热稳定性能.

\section{2 实验部分}

\section{1 主要原料}

ODOPB (10-(2,5-二羟基苯基)-10-氢-9-氧杂-10-磷 杂菲-10-氧化物), 化学纯, 广东惠州盛世达科技有限公 司; 对甲基苯酚, 分析纯, 北京百灵威科技有限公司; 三 氯甲烷 $\left(\mathrm{CHCl}_{3}\right)$ ，分析纯，天津市富宇精细化工有限公 司; 环己烷，分析纯，成都市科龙化工试剂厂; 氯化铝 $\left(\mathrm{AlCl}_{3}\right)$ 、间苯二胺 $(\mathrm{PDA})$, 分析纯, 天津市光复精细化 工研究所; 聚磷酸铵(APP), 分析纯, 什邡市太丰新型阻 燃剂有限公司; 三氯氧磷 $\left(\mathrm{POCl}_{3}\right)$ 、蒙脱土(MMT), 分 析纯, 国药集团化学试剂有限公司; 氯化钙, 分析纯, 西 陇化工股份有限公司; 环氧树脂(EP), 工业品, 蓝星化 工新材料股份有限公司.

\section{2 阻然剂的制备}

\subsubsection{PPTP的制备}

二氯化磷酸对甲苯酯（PPTP）的合成过程如下 ${ }^{[14]}$ :
首先在 $100 \mathrm{~mL}$ 圆底烧瓶中加入三氯氧磷 $30.7 \mathrm{~g}$ $(0.2 \mathrm{~mol}), 88^{\circ} \mathrm{C}$ 油浴恒温加热, 然后在 $30 \mathrm{~min}$ 内缓慢滴

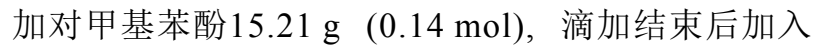
$0.07 \mathrm{~g}$ (0.0006 mol) $\mathrm{CaCl}_{2}$, 最后将反应温度升至 $92^{\circ} \mathrm{C}$, 不断摚拌反应 $2 \mathrm{~h}$, 将得到的混合物减压蒸馏, 得到目 标产物PPTP, 产率约为 $90 \%$.

\subsubsection{POTP的制备}

POTP的合成反应如图1所示，具体过程如下 ${ }^{[15]}$ : 取 $250 \mathrm{~mL}$ 三颈烧瓶，分别向其中加入PPTP (28 g, $0.13 \mathrm{~mol}), \mathrm{AlCl}_{3}(0.2 \mathrm{~g}, 0.001 \mathrm{~mol}$ )和ODOPB (40.6 g, $0.13 \mathrm{~mol}), \mathrm{N}_{2}$ 氛围下升温至 $60^{\circ} \mathrm{C}$, 不断搅拌反应 $30 \mathrm{~min}$, 升温至 $90^{\circ} \mathrm{C}$ 反应 $1 \mathrm{~h}$ ，继续将温度升到 $140^{\circ} \mathrm{C}$ 反应 $1 \mathrm{~h}$, 然后升至 $200^{\circ} \mathrm{C}$ 反应至产物变黏稠后停止，冷却到室 温, 用粉碎机将其粉碎为白色固体粉末，再用适量的 氯仿进行溶解, 待溶解完全后加入再适量的环己烷沉 淀，抽滤后置于真空烘箱中，在 $60^{\circ} \mathrm{C}$ 温度下干燥 $8 \mathrm{~h}$ 即 可得到产物POTP, 产率约为 $86 \%$.

\section{3 阻燃EP样品的制备}

阻燃EP复合材料采用热固化工艺制备. 先用丙酮 对环氧树脂进行稀释, 然后加入阻燃剂搅拌均匀, 置于 $80^{\circ} \mathrm{C}$ 真空烘箱中除去溶剂, 最后加入固化剂间苯二胺 (PDA), 继续搅拌直至混合均匀, 真空脱泡 $10 \mathrm{~min}$, 将 混合物倒入预热的特定尺寸聚四氟乙烯模具中，固化 条件为 $80^{\circ} \mathrm{C} / 2 \mathrm{~h}, 100^{\circ} \mathrm{C} / 2 \mathrm{~h}$ 和 $120^{\circ} \mathrm{C} / 2 \mathrm{~h}$, 将所制备的固 化物样条取出即可.

\section{4 测试与表征}

采用傅里叶变换红外光谱仪(FTIR, Nicolet iS5, Thermo Scientific, 美国)测定红外谱图, 样品制备采用 $\mathrm{KBr}$ 压片法, 测试范围为500 4000 $\mathrm{cm}^{-1}$.

采用瑞士布鲁克Bruker Advanced 600 (600MHz) 型核磁共振仪, 用気代氯仿为溶剂，测定 ${ }^{1} \mathrm{H}-\mathrm{NMR}$ 、

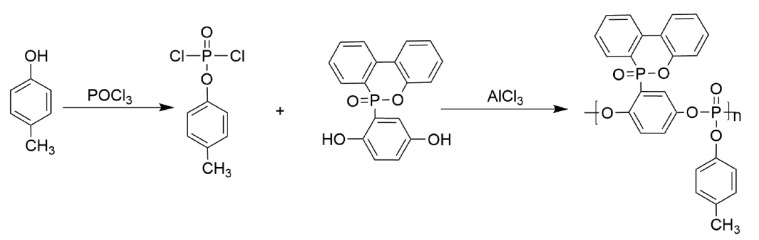

图 1 POTP合成路线

Figure 1 Synthesis route of POTP 
${ }^{13} \mathrm{C}-\mathrm{NMR}$ 谱图.

热重(TGA)分析测试采用德国NETZSCHSTA 409 $\mathrm{PC} / \mathrm{PG}$ 热重分析仪, 氮气氛围, 氮气流量为 $20 \mathrm{~mL} / \mathrm{min}$, 升温速率 $20^{\circ} \mathrm{C} / \mathrm{min}$, 升温范围 $40 \sim 700^{\circ} \mathrm{C}$.

氧指数测试(LOI) (JF-5, 南京畑雷仪器设备有限 公司)按照ASTMD-2863测试标准进行测试, 试样尺寸 为 $120 \mathrm{~mm} \times 6.5 \mathrm{~mm} \times 3.2 \mathrm{~mm}$.

垂直燃烧测试(UL-94)采用CZF-6型水平燃烧测定 仪(南京市江宁区分析仪器厂), 按照ASTMD3801标准 进行测试, 试样尺寸为 $120 \mathrm{~mm} \times 13 \mathrm{~mm} \times 3.2 \mathrm{~mm}$.

锥形量热测试(cone calorimetry (CONE)) (FTT10007, 英国Fire Testing Technology公司)按标ISO5660-1标准 进行雉形量热测定, 热流量为 $35 \mathrm{~kW} / \mathrm{m}^{2}$ 样品尺寸为 $100 \mathrm{~mm} \times 100 \mathrm{~mm} \times 1.2 \mathrm{~mm}$.

采用扫描电子显微镜 $(\mathrm{SEM}, \mathrm{SM}-7500 \mathrm{~F}$ 型, 日本 JEOL公司)进行断面形貌分析, 加速电压为 $5 \mathrm{kV}$, 真空 喷金处理.

\section{3 结果与讨论}

\subsection{POTP的结构表征}

采用FTIR对PPTP和POTP进行了结构表征, 通 过 ${ }^{31} \mathrm{P}-\mathrm{NMR} 、{ }^{1} \mathrm{H}-\mathrm{NMR}$ 和 ${ }^{13} \mathrm{C}-\mathrm{NMR}$ 进一步对POTP的结 构进行表征. 图2为PPTP和POTP的红外光谱(FTIR), 由 曲线 $\mathrm{a}$ 可知, 在 $820 \mathrm{~cm}^{-1}$ 处出现 $\mathrm{P}-\mathrm{Cl}$ 的伸缩振动吸收峰, $1164 \mathrm{~cm}^{-1}$ 处为 $\mathrm{P}-\mathrm{O}-\mathrm{Ph}$ 的伸缩振动吸收峰, $1365 \mathrm{~cm}^{-1}$ 为 $\mathrm{P}=\mathrm{O}$ 的强弯曲振动吸收峰, $3035 \mathrm{~cm}^{-1}$ 为苯环 $\mathrm{C}-\mathrm{H}$ 键的 伸缩振动吸收峰. 由曲线 $\mathrm{b}$ 可以看出, $1024 \mathrm{~cm}^{-1}$ 和 $1167 \mathrm{~cm}^{-1}(\mathrm{P}-\mathrm{O}-\mathrm{Ph}), 1327 \mathrm{~cm}^{-1}(\mathrm{P}=\mathrm{O}), 1545 \mathrm{~cm}^{-1}(\mathrm{P}-\mathrm{C})$, 在 $820 \mathrm{~cm}^{-1}$ 处 $\mathrm{P}-\mathrm{Cl}$ 的伸缩振动吸收峰消失, 说明所合 成的目标产物中不存在PPTP. POTP 的 ${ }^{31}$ P-NMR测试如

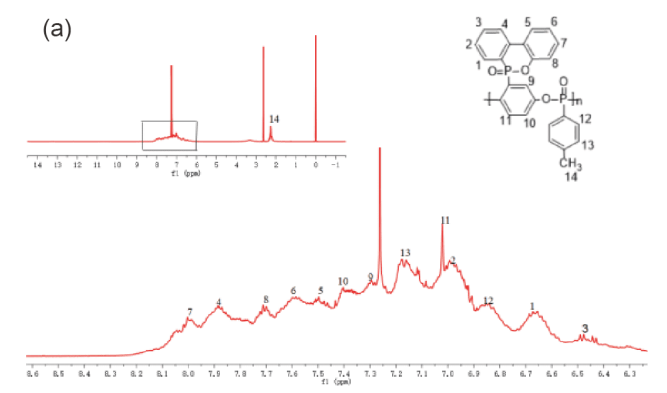

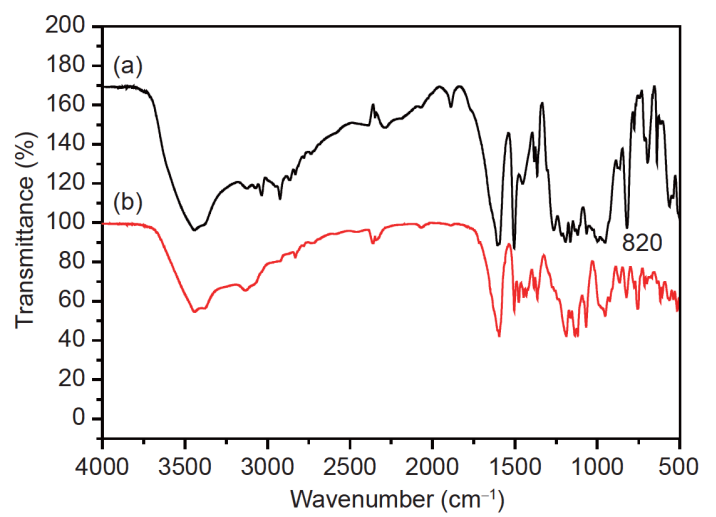

图 2 PPTP(a)和POTP(b)的红外光谱(网络版彩图)

Figure 2 FTIR spectra of PPTP(a) and POTP(b) (color online).

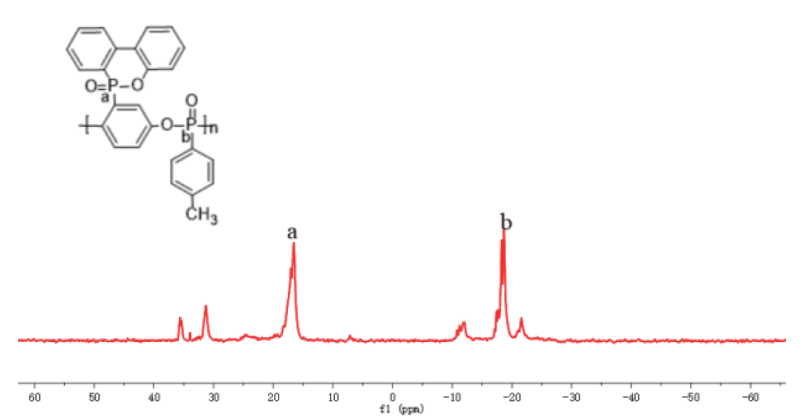

图 3 POTP的 ${ }^{31} \mathrm{P}-\mathrm{NMR}$ 谱图(网络版彩图)

Figure $3{ }^{31} \mathrm{P}-\mathrm{NMR}$ spectrum of POTP (color online).

图3所示, POTP中有两种不同化学环境的磷原子, 其中 主链上的磷是磷酸三酯, 出峰位置在 $-18 \mathrm{ppm}$ 左右, 侧 链上的磷是一个环状的二取代膦酯单酯, 出峰位置大 约在 17 ppm左右. POTP的 ${ }^{1} \mathrm{H}-\mathrm{NMR}$ 和 ${ }^{13} \mathrm{C}-\mathrm{NHR}$ 如图4所 示. ${ }^{1} \mathrm{H}-\mathrm{NMR}\left(\mathrm{CHCl}_{3}, 400 \mathrm{MHz}, \delta\right): 7.87(\mathrm{~s}, 1 \mathrm{H}), 7.41(\mathrm{~s}$, $1 \mathrm{H}), 7.51(\mathrm{~s}, 1 \mathrm{H}), 8.02(\mathrm{~s}, 1 \mathrm{H}), 7.70(\mathrm{~s}, 1 \mathrm{H}), 7.09(\mathrm{~s}, 1 \mathrm{H})$, $7.26(\mathrm{~s}, 1 \mathrm{H}), 7.01(\mathrm{~s}, 1 \mathrm{H}), 6.50(\mathrm{~s}, 1 \mathrm{H}), 6.89(\mathrm{~s}, 1 \mathrm{H}), 6.65$ (s, 1H), $6.83(\mathrm{~s}, 2 \mathrm{H}), 7.07(\mathrm{~s}, 2 \mathrm{H}), 2.26(\mathrm{~s}, 1 \mathrm{H}) ;{ }^{13} \mathrm{C}-\mathrm{NMR}$

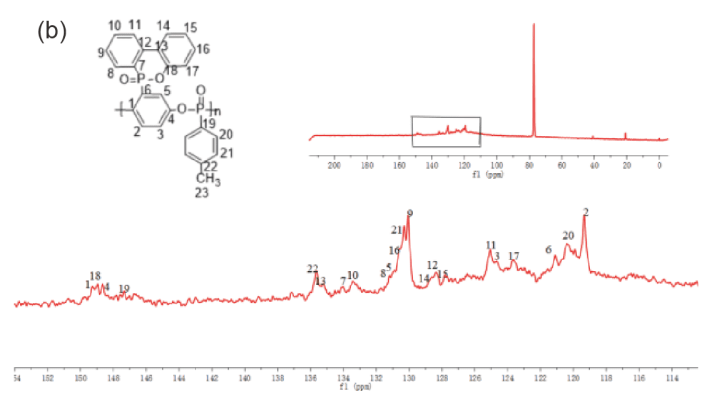

图 4 POTP的 ${ }^{1} \mathrm{H}-\mathrm{NMR}(\mathrm{a})$ 和 ${ }^{13} \mathrm{C}-\mathrm{NMR}$ (b)谱图(网络版彩图) Figure 4 The ${ }^{1} \mathrm{H}-\mathrm{NMR}$ (a) and ${ }^{13} \mathrm{C}-\mathrm{NMR}$ (b) of POTP (color online). 
$\left(\mathrm{CHCl}_{3}, 400 \mathrm{MHz}, \delta\right): 148.9$ (s, 1C), 119.3 (s, 1C), 124.7 (s, 1C), 148.7 (s, 1C), 130.9 (s, 1C), 121.1 (s, 1C), 133.4 (s, 1C), $131.2(\mathrm{~s}, 1 \mathrm{C}), 130(\mathrm{~s}, 1 \mathrm{C}), 134(\mathrm{~s}, 1 \mathrm{C}), 125.1(\mathrm{~s}$, 1C), $128.4(\mathrm{~s}, 1 \mathrm{C}), 135.2(\mathrm{~s}, 1 \mathrm{C}), 128.6(\mathrm{~s}, 1 \mathrm{C}), 127.8(\mathrm{~s}$, 1C), 130.3 (s, 1C), 123.6 (s, 1C), $149.26(\mathrm{~s}, 1 \mathrm{C}), 147.3$ (s, 1C), 120.4 (s, 1C), 130.6 (s, 1C), 135.6 (s, 1C), 20.7 (s, $1 \mathrm{C})$. 由上叙结果可知，所合成的目标产物的 ${ }^{1} \mathrm{H}-\mathrm{NMR}$ 和 ${ }^{13} \mathrm{C}-\mathrm{NMR}$ 的化学位移与预期的POTP的结构相符, 说 明POTP成功合成.

\section{2 阻燃EP的阻燃性能分析}

\subsection{1 阻燃EP的LOI和UL-94的测试}

通常用极限氧指数(LOI)和垂直燃烧(UL-94)来评 估材料的阻燃性，阻燃效率(EFF)被定义为阻燃系统中 单位质量阻然元素对LOI值的贡献。在POTP/MMT/ $\mathrm{APP} / \mathrm{EP}$ 复配体系中, MMT的均添加量均为 $1 \%$, POTP 和 APP的添加比例为 $1: 2$. 表 1 列出了阻燃EP的配方及 LOI测试、UL-94测试及EFF的数据. 由表中数据可以 看出, EP-0\#的LOI值为 $19.8 \%$, 阻燃EP的LOI值较EP0\#的高. 随着阻燃剂POTP/MMT/APP添加量的不断增 加, 阻燃EP的LOI值逐渐增加, 而EFF值先增大后减小, 当阻燃剂的添加量为 $7 \%$ 时，阻燃EP的LOI值达到 $25.6 \%$, UL-94阻燃级别可达到V-0级; 当阻燃剂的加入 量为 $9 \%$ 时, 阻燃EP的LOI值可达到 $27.6 \%$. 这说明阻燃 剂POTP/MMT/APP的加入改变了材料的燃烧行为, 有 效提高了环氧树脂的LOI值.

\subsection{2 阻燃EP的雉形量热分析}

图5给出了纯EP及阻燃 $\mathrm{EP}$ 复合材料的热释放速率 (HRR)、热释放总量(THR)、生烟速率(SPR)和烟释放 总量(TSR)等特征曲线, 其相应数据列于表 2 .
点火时间(TTI) 和材料的防火性能指数(FPI)值是 描述复合材料燃烧特性的重要手段，通常FPI值越小， 火灾风险越大 ${ }^{[16]}$. 从表 2 可以看出, 虽然阻燃 $E P$ 复合材 料的TTI值随着阻燃剂POTP/MMT/APP含量的增加而 降低，但是FPI值随阻燃剂的增加先减小后增大，因此 加入适量的阻燃剂后可以降低EP复合材料的火灾风 险性．热释放速率(HRR)通常是表征火灾强度的最重 要的性能参数, 也是评价材料潜在火灾危险性的重要 指标, 其值越大表明火灾危险性越高 ${ }^{[17]}$. 结合图 $5(\mathrm{a}, \mathrm{b})$ 和表2可知, EP-1\#的最大热释放速率(Pk-HRR)高于 EP-0\#, 这主要源于阻燃剂的添加量较少，导致燃烧生 成的炭层不稳定，高温条件下炭层被破坏促使EP再次 燃烧，使得复合材料的Pk-HRR值增大; 随着阻燃剂 POTP/MMT/APP填充量的增加，阻燃EP复合材料的 $\mathrm{Pk}-\mathrm{HR} R$ 值和THR值均逐渐减小，当POTP/MMT/APP 填充量为 $9 \%$ 时, Pk-HRR值下降至 $496.2 \mathrm{~kW} / \mathrm{m}^{2}$, THR值下 降至 $64.2 \mathrm{MJ} / \mathrm{m}^{2}$, 分别降低了 $50.1 \%$ 和 $27.4 \%$, 降低的趋 势明显. 说明阻燃剂POTP/MMT/APP对EP基材具有良 好的阻燃效果，能有效降低EP基材的HRR 和THR值， 降低EP基材在火灾中存在的潜在威胁. 复合材料良好 的阻燃效果可能来自于: 一方面, 阻燃剂分解产生磷酸 和多聚磷酸催化EP基材成炭, 所形成的炭层覆盖在EP 基材表面阻止热量的传递，同时还抑制可燃气体从内 部逸出，从而对材料起到很好的保护作用 ${ }^{[18]}$; 另一方 面，阻燃剂通过释放·PO来捕获气态的· $\mathrm{H}$ 和· $\mathrm{OH}$ ，诱导 炭层的形成, 延缓EP基材的降解和燃烧过程 ${ }^{[19]}$.

烟释放总量(TSR)和生烟速率(SPR)是评价材料潜 在火灾发生时烟气危险的重要参数. 结合图5(c)和(d) 可以看出，在 $130 \mathrm{~s}$ 时，PE-1\#出现SPR峰值较EP-0\#高, $125 \mathrm{~s}$ 时, EP-4\#出现SPR峰值; 阻燃EP的TSR和SPR值 均低于EP-0\#，随着阻燃剂POTP/MMT/APP添加量的

表 1 阻燃EP复合材料的配方及LOI/UL-94数据

Table 1 Formulation and LOI/UL-94 data of flame retardant EP composites

\begin{tabular}{|c|c|c|c|c|c|c|c|c|}
\hline \multirow{2}{*}{ Sample } & \multicolumn{5}{|c|}{ Mass fraction (\%) } & \multirow{2}{*}{ LOI $(\%)$} & \multirow{2}{*}{ UL-94 } & \multirow{2}{*}{ EFF } \\
\hline & EP & POTP & MMT & APP & m-PDA & & & \\
\hline EP-0\# & 91 & 0 & 0 & 0 & 9 & 19.8 & N.R. & 1 \\
\hline EP-1\# & 88 & 0.67 & 1 & 1.33 & 9 & 23.1 & N.R. & 0.66 \\
\hline EP-2\# & 86 & 1.33 & 1 & 2.67 & 9 & 24.9 & V-1 & 1.02 \\
\hline EP-3\# & 84 & 2 & 1 & 4 & 9 & 25.6 & $\mathrm{~V}-0$ & 0.83 \\
\hline EP-4\# & 82 & 2.67 & 1 & 5.33 & 9 & 27.6 & $\mathrm{~V}-0$ & 0.83 \\
\hline
\end{tabular}



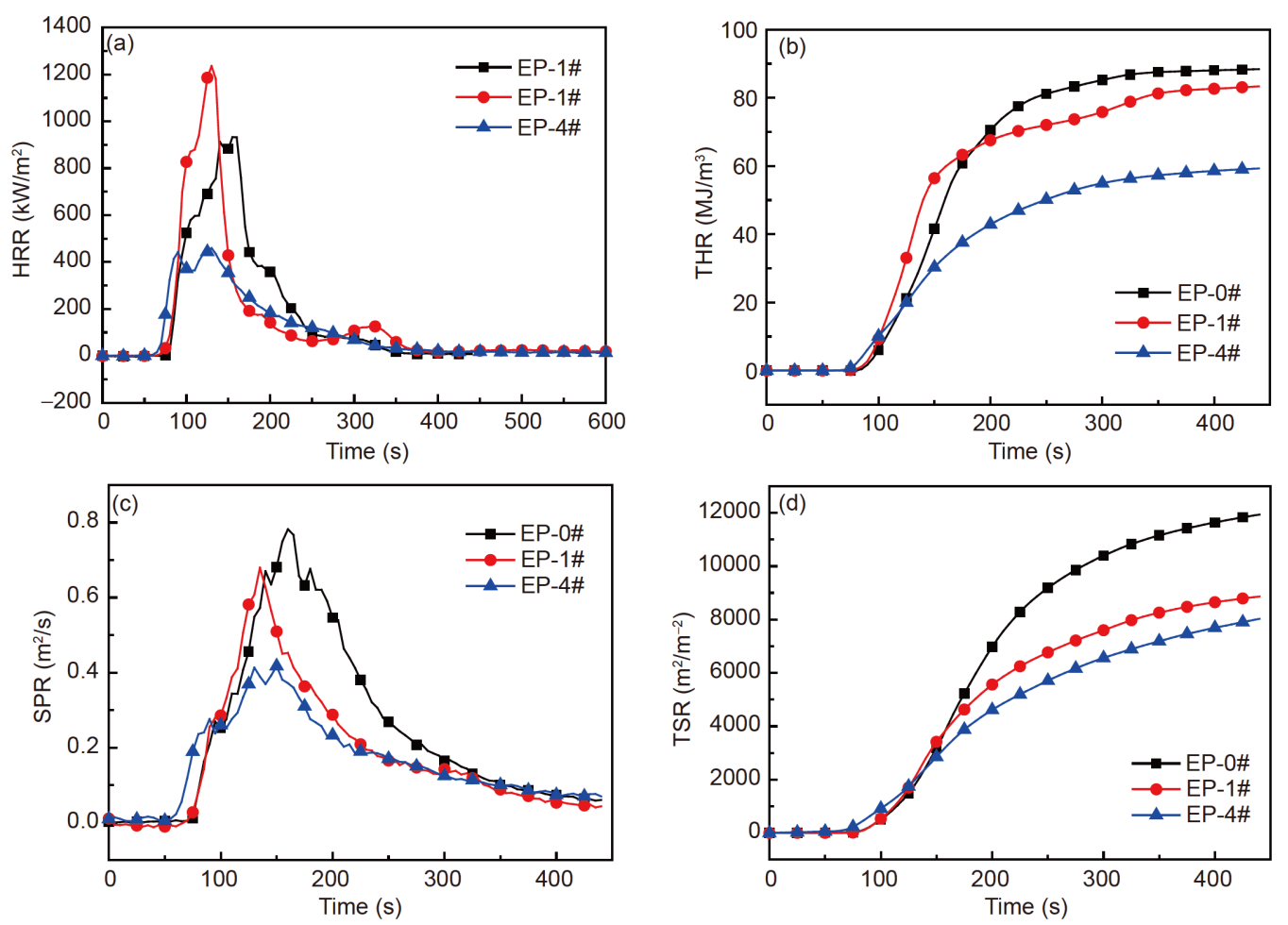

图 5 EP及其阻燃复合材料的雉形量热曲线: (a) HHR, (b) THR, (c) SPR, (d) TSR (网络版彩图)

Figure 5 Cone calorimetry curves of EP and its flame retardant composites: (a) HHR, (b) THR, (c) SPR, (d) TSR (color online).

表 2 POTP/APP/MMT/EP的雉形量热法数据

Table 2 Data from cone calorimetry of POTP/APP/MMT/EP

\begin{tabular}{cccccccc}
\hline Sample & TTI $(\mathrm{s})$ & Pk-HRR $\left(\mathrm{kW} / \mathrm{m}^{2}\right)$ & FPI $\left(\mathrm{sm}^{2} / \mathrm{kW}\right)$ & Av-HRR $\left(\mathrm{kW} / \mathrm{m}^{2}\right)$ & THR $\left(\mathrm{MJ} / \mathrm{m}^{2}\right)$ & TSP $\left(\mathrm{m}^{3}\right)$ & Mass loss $(\mathrm{g})$ \\
\hline EP-0\# & 70 & 993.1 & 0.075 & 238.9 & 88.4 & 105.3 & 91.9 \\
EP-1\# & 60 & 1235.7 & 0.048 & 149.9 & 87.7 & 83.3 & 87.4 \\
EP-4\# & 55 & 496.2 & 0.11 & 101.2 & 64.2 & 75.4 & 77.7 \\
\hline
\end{tabular}

增加，TSR、SPR曲线逐渐趋于平缓. 由于燃烧刚开始 时阻燃剂分解产物能促进环氧树脂基体的降解，导致 烟提前排放，随着燃烧的进行环氧树脂基体表面形成 致密、连续的炭层，可以阻止热量的传递，抑制可燃 气体的生成，从而抑制燃烧热解反应的强度，减少烟 释放总量 ${ }^{[13]}$.

\subsection{3 阻然EP的残炭测试}

为了更好地分析POTP/MMT/APP对EP的阻燃机 理, 对雉形量热测试后的炭层表面形貌进行了分析. 图 6给出了纯EP和阻燃EP复合材料燃烧后残炭的形貌 图. 由图6(a)、(b)和(c)可以看出，EP-0\#燃烧过后几乎
没有残余物, 随着阻燃剂加入比例的增加, 残炭量逐渐 增多，同时还可以看到产生的炭层发生膨胀现象; EP1 \#和EP-4\#试样燃烧后的厚度分别达到了 $2.8 \mathrm{~cm}$ 和 $5.6 \mathrm{~cm}$, 说明阻燃剂有效地提高了 $\mathrm{EP}$ 基材的成炭性能. 一方面，阻燃剂分解产生磷酸和聚磷酸等充当酸源和 炭源的作用催化EP基材表面成炭，使燃烧后阻燃EP的 残炭量增加 ${ }^{[20,21]}$; 另一方面，MMT在受热情况下产生 的霍夫曼效应和硅酸盐陶瓷结构利于膨胀阻燃剂的交 联成炭和更优质炭层的形成 ${ }^{[22]}$. 同时结合图6(d) 可以 看到EP-0\#产生的炭层是不连续的，此外还有空洞产 生，为可燃挥发物进入提供了气态通道，所以不能起 到很好的屏蔽作用，导致EP-0\#易燃烧. 结合图6(e)和 

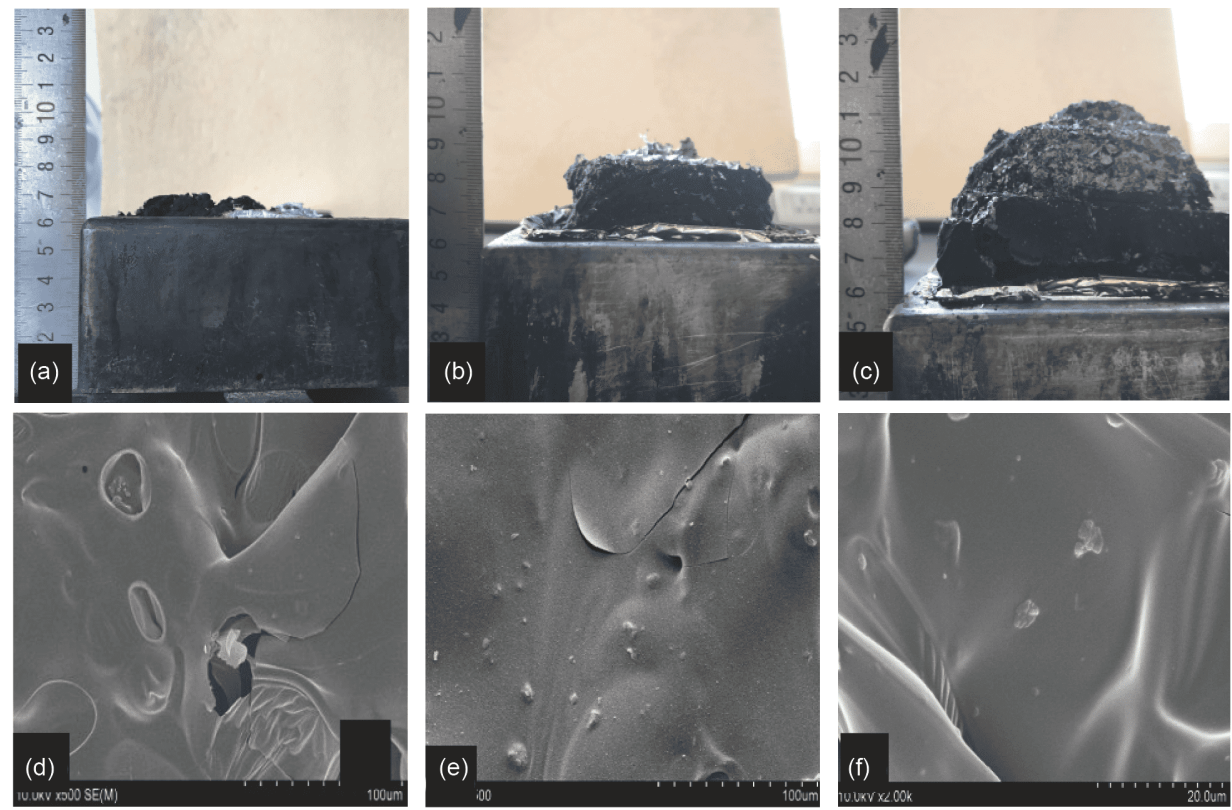

图 6 锥形量热测试后的POTP/APP/MMT/EP照片(a c)及SEM图谱(d f). (a, d) EP-0\#, (b, e) EP-1\#, (c, f) EP-4\# (网络版彩图) Figure 6 Photos $(\mathrm{a}-\mathrm{c})$ and SEM images $(\mathrm{d}-\mathrm{f})$ of POTP/APP/MMT/EP after CONE measurement. (a, d) EP-0\#, (b, e) EP-1\#, (c, f) EP-4\# (color online).

(f)可以看出, 加入阻燃剂POTP/MMT/APP后EP燃烧产 生了更加连续且致密的炭层，这种结构可以有效地阻 止可降解物质的释放，阻止可燃性气体向火焰区域迁 移，从而阻止火焰的蔓延和传播，防止基材继续燃烧， 增强EP基材的阻燃性能 ${ }^{[23]}$.

基于上述的燃烧行为，本文提出了阻燃剂PODOPTP/MMT/APP阻燃EP的机理, 如图7所示. 在燃烧 过程中, 当温度升到 $320^{\circ} \mathrm{C}$ 以上, $\mathrm{POTP}$ 分解产生的 $\cdot \mathrm{PO}$ 可以捕获气态的 $\cdot \mathrm{H}$ 和 $\cdot \mathrm{OH}$ ，使高分子聚合物燃烧过程 中的链式反应终止，发挥气相阻燃作用，从而减缓燃 烧; APP分解产生磷酸和聚磷酸等充当酸源，催化EP 脱水形成致密连续炭层，保护底层基质不受外部热辐 射的破坏及阻止可燃气体从内部逸出，当温度升高进 一步分解产生 $\mathrm{NH}_{3}$ 等可以作为惰性气体稀释氧气的浓 度；MMT不但可以提高DOPOTP和APP与EP的相容 性, 而且MMT在受热情况下产生陶瓷结构的硅酸盐有 利于促进阻燃剂的交联成炭, 形成优质炭层.

\section{3 阻燃剂对EP的热性能的影响}

为了研究阻燃剂POTP/MMT/APP对EP热稳定性 的影响，在氮气氛围下对纯EP和阻燃EP进行了 $\mathrm{TG}$ 测 试. 图8为复合材料的 TG及DTG曲线，其具体数据列

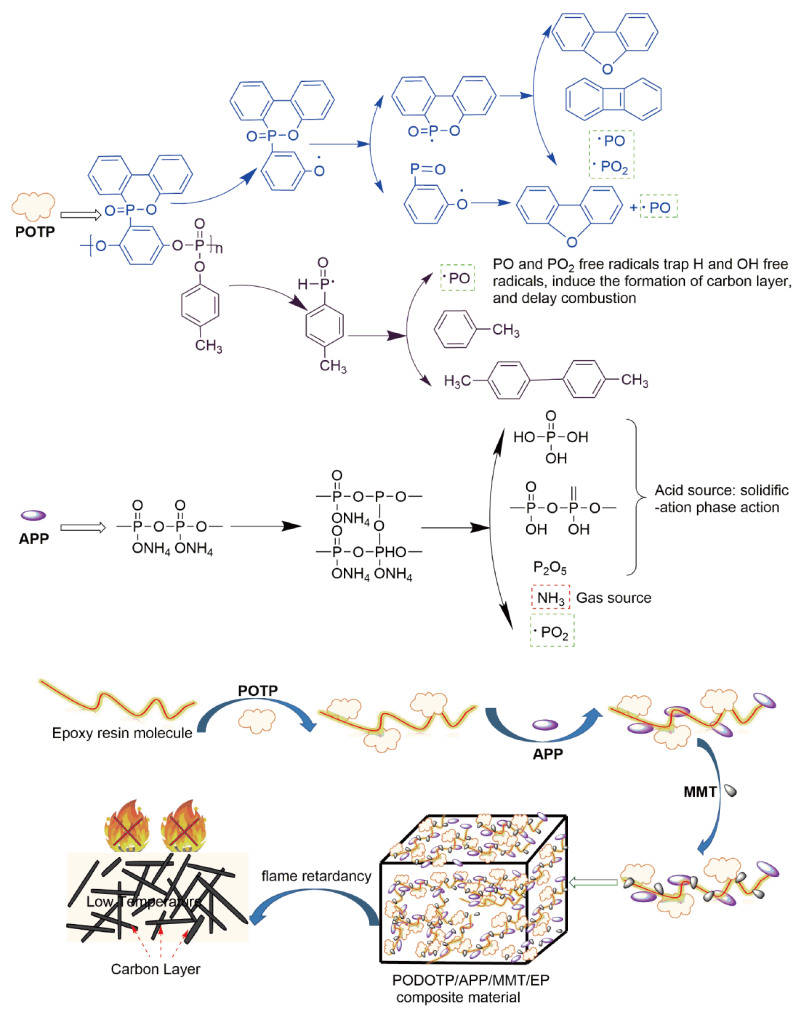

图 7 POTP/MMT/APP-EP阻燃机理示意图(网络版彩图)

Figure 7 Schematic illustration of the flame-retardation mechanism of POTP/MMT/APP-EP (color online) 

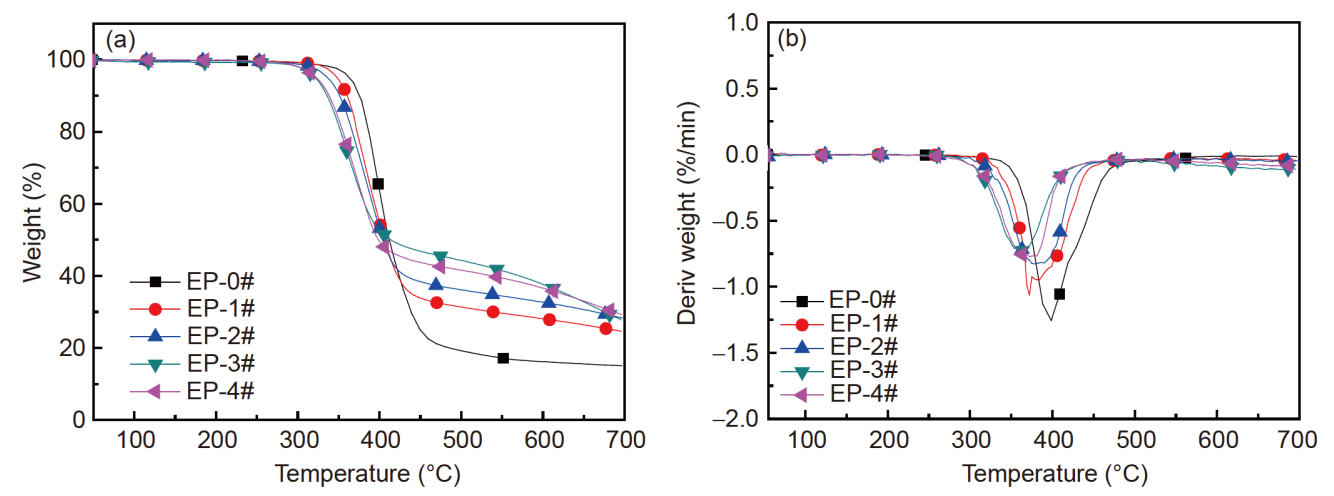

图 $8 \mathrm{POTP} / \mathrm{APP} / \mathrm{MMT} / \mathrm{EP}$ 的TG (a)和DTG (b)曲线(网络版彩图)

Figure 8 TG (a) and DTG (b) curves of POTP/APP/MMT/EP (color online).

表 3 POTP/APP/MMT/EP的TG和DTG数据

Table 3 TG and DTG data of POTP/APP/MMT/EP

\begin{tabular}{cccccc}
\hline Sample & $T_{5 \%}\left({ }^{\circ} \mathrm{C}\right)$ & $T_{10 \%}\left({ }^{\circ} \mathrm{C}\right)$ & $T_{50 \%}\left({ }^{\circ} \mathrm{C}\right)$ & $T_{\max }\left({ }^{\circ} \mathrm{C}\right)$ & $R_{7000^{\circ} \mathrm{C}}(\%)$ \\
\hline EP-0\# & 357 & 378 & 408 & 397 & 15.03 \\
EP-1\# & 348 & 360 & 405 & 372 & 24.65 \\
EP-2\# & 336 & 351 & 404 & 380 & 28.22 \\
EP-3\# & 321 & 336 & 410 & 363 & 27.81 \\
EP-4\# & 321 & 329 & 399 & 373 & 29.32 \\
\hline
\end{tabular}

于表3．其中重量损失 $5 \%$ 时的温度定义为初始分解温 度, $T_{\max }$ 定义为最大失重速率时的温度. 结合图 $8(\mathrm{a})$ 、 (b)和表 3 可以看出, 阻燃 $\mathrm{EP}$ 复合材料的 $T_{5 \%}$ 和 $T_{\max }$ 均低 于EP-0\#, 且随着阻燃剂POTP/MMT/APP含量的增加 其 $T_{5 \%}$ 和 $T_{\max }$ 逐渐降低. 这归因于阻燃剂不稳定, 在较 低温度下分解产生磷酸、聚磷酸及可发生酯化的多元 醇等促进了 $\mathrm{EP}$ 基材的降解，导致阻燃 $\mathrm{EP}$ 复合材料的 $T_{5 \%}$ 和 $T_{\text {max }}$ 提前 ${ }^{[24]}$. 由表 3 可知, $700{ }^{\circ} \mathrm{C}$ 时阻燃 $\mathrm{EP}$ 的残炭 量均比EP-0\#高, 最高的EP-4\#残炭量可达到 $29.32 \%$, 比EP-0\#高出 $14.29 \%$, 说明阻燃剂POTP/MMT/APP的 加入有效改善了EP的热降解行为，使其具有更好的成 炭效果. 由于阻燃剂分解产物可充当酸源促进EP基材 的自身交联作用形成具有高热稳定性的炭层，保护底 层EP基体不被进一步降解, 因此导致阻燃 $\mathrm{EP}$ 的残炭量 增加, 说明阻燃剂的加入能有效改善EP的阻燃性能及 热稳定性.

\section{4 结论}

（1）本文合成了DOPO衍生物POTP，并采用 FIIR、 ${ }^{31} \mathrm{P}-\mathrm{NMR} 、{ }^{1} \mathrm{H}-\mathrm{NMR}$ 和 ${ }^{13} \mathrm{C}-\mathrm{NMR}$ 对其进行了结 构表征; (2) 将POTP与MMT及APP复配对EP进行阻燃 改性, 当阻燃剂添加量为 $7 \%$ 时, 阻燃EP复合材料UL94 测试可达 V-0 级, LOI值为 $25.6 \%$; 当添加阻燃剂为 $9 \%$ 时, 阻燃EP复合材料的LOI值可达到 $27.6 \%$. 阻燃剂 的加入使 EP初始分解温度有所降低, 但其最大热释放 速率下降了 $50.1 \%$, 热释放总量下降了 $27.4 \%$, 残炭量 高达 $29.32 \%$, 阻燃剂有效地提高了EP的阻燃性能和热 性能. (3) POTP/MMT/APP阻燃剂能促进EP燃烧时基 材表面的成炭能力, 在其表面形成连续致密的炭层, 这 种结构能有效地阻碍材料的热量传递, 抑制可燃气体 的生成和EP的生烟速率, 提高EP的热性能和阻燃 性能.

\section{参考文献}

1 Ma SQ, Liu XQ, Jiang YH, Fan LB, Feng JX, Zhu J. Sci China Chem, 2013, 57: 379-388 
2 Jiang GZ, Pickering SJ, Lester EH, Warrior NA. Industrial \& Engineering Chemistry Research, 2010, 49: 4535-4541

3 Mustata FR, Tudorachi N. Industrial \& Engineering Chemistry Research, 2010, 49(24): 12414-12422

4 Tang RG, Huang ZG. Bulletin of Science and Technology, 2012, 28: 129-132 (in Chinese) [唐若谷, 黄兆阁. 科技通报, 2012, 28: 129-132]

5 Tian N, Gong J, Wen X, Yao K, Tang T. RSC Adv, 2014, 4: 17607-17614

6 Luo H, Zhou F, Yang Y, Cao X, Cai X. J Therm Anal Calorim, 2017, 132: 483-491

7 Spontón M, Ronda JC, Galià M, Cádiz V. J Polym Sci A Polym Chem, 2007, 45: 2142-2151

8 Shieh JY, Wang CS. J Polym Sci A Polym Chem, 2002, 40: 369-378

9 Tang S, Qian L, Qiu Y, Dong Y. Polym Degradation Stability, 2018, 153: 210-219

10 Wu H, Hu R, Zeng B, Yang L, Chen T, Zheng W, Liu X, Luo W, Dai L. RSC Adv, 2018, 8: 37631-37642

11 Perret B, Schartel B, Stöß K, Ciesielski M, Diederichs J, Döring M, Krämer J, Altstädt V. Eur Polym J, 2011, 47: 1081-1089

12 Zhang Q, Yang S, Wang J, Cheng J, Zhang Q, Ding G, Hu Y, Huo S. Polym Degradation Stability, 2019, 167: 10-20

13 Xu MJ, Xu GR, Leng Y, Li B. Polym Degradation Stability, 2016, 123: 105-114

14 Huo ZL. Hebei Chemical Engineering and Industry, 2006, 6: 19-21 (in Chinese) [霍竹林. 河北化工, 2006, 6: 19-21]

15 Ban DM, Liu JP. Chemical Journal of Chinese Universities-Chinese, 2013, 34: 1284-1287

16 Hirschler MM. Polym Adv Technol, 2008, 19: 521-529

17 Chen SM, Lai F, Li P, Gong W, Fu H, Yin XG, Ban DM. Acta Polymerica Sinica, 2017, 8: 1358-1365 (in Chinese) [陈仕梅, 来方, 李需, 龚维, 付 海, 尹晓刚, 班大明. 高分子学报, 2017, 8: 1358-1365]

18 Fang YC, Zhou X, Xing Z, Wu YR. Journal of Applied Polymer Science, 2017, 134(12): 44639

19 Wang X, Hu Y, Song L, Yang H, Xing W, Lu H. Prog Org Coatings, 2011, 71: 72-82

20 Bourbigot S, Bras ML, Duquesne S, Rochery M. Macromolecular Materials and Engineering, 2004, 289: 99-511

21 Qian XX, Song L, Hu Y, Richard KK, Chen LJ, Guo YQ, Hong NN, Jiang SH. Industrial \& Engineering Chemistry Research, 2011, 50: 18811892

22 Lu LG, Chen YH, Wang SH, Yang SS, Dong XL. Journal of Materials Research, 2014, 28: 455-461 (in Chinese) [卢林刚, 陈英辉, 王舒衡, 杨守 生, 董希琳.材料研究学报, 2014, 28: 455-461]

23 Chen X, Jiao C, Li S, Sun J. J Polym Res, 2011, 18: 2229-2237

24 Ran SY, Xu YY, Guo ZH, Fang ZP. Chemical Journal of Chinese Universities, 2013, 34: 467-473 (in Chinese) [舟诗雅, 许远远, 郭正虹, 方征 平. 高等学校化学学报, 2013, 34: 467-473] 


\title{
Effect of polyphosphate flame retardant compounded montmorillonite and ammonium polyphosphate on flame retardancy of epoxy resin
}

\author{
Ji Yang, Yonghang Zhang, Juanjuan Fan, Yang Min, Daming Ban \\ School of Chemistry and Materials Science, Guizhou Normal University, Guiyang 550001, China \\ *Corresponding author (email: bdaming@gznu.edu.cn)
}

\begin{abstract}
A new polyphosphate flame retardant, polyphosphate-2-10-hydrogen-9-oxa-10-phosphaphenanthrene-10oxide hydroquinone p-tolyl ester (POTP), was successful synthesized by p-methyl dichlorophosphate acid phenyl ester and 10-(2,5-dihydroxyphenyl)-10-hydro-9-oxa-10-phosphaphenanthrene-10-oxide (ODOPB). The chemical structure was characterized by Fourier transform infrared spectroscopy (FTIR) and nuclear magnetic resonance $\left({ }^{31} \mathrm{P}-\mathrm{NMR},{ }^{1} \mathrm{~N}-\right.$ NMR and ${ }^{13} \mathrm{C}-\mathrm{NMR}$ ). The POTP with ammonium polyphosphate (APP) and montmorillonite (MMT) was added to epoxy resin (EP) for Flame retardant modified. The flame retardancy and thermal performance of epoxy composites were investigated by vertical burning (UL-94), limiting oxygen index (LOI), thermal weight loss (TGA), cone calorimetry (CONE) and scanning electron microscope (SEM). The results showed that epoxy composites obtained a UL-94 V-0 grade when 5\% flame retardant added. When the added amount of flame retardant was increased to $9 \%$, a higher LOI value of $27.6 \%$ can be achieved. The cone test showed that the residual carbon content was as high as $29 \%$, and the peak of heat release rate (Pk-HHR) and total heat release (THR) decreased by $41.7 \%$ and $31.1 \%$, respectively. The thermal stability and flame retardant properties of flame retardant EP at high temperatures have been improved. The morphology of residual carbon after the CONE test shows that flame retardant EP forms a more stable and dense expanded carbon layer at high temperature. Its carbon structure can isolate the exchange of combustible gas and air, and effectively inhibit the release of toxic gases in the flue gas.
\end{abstract}

Keywords: epoxy resin, montmorillonite, ammonium polyphosphate, flame retardancy

doi: $10.1360 /$ SSC-2020-0010 